\title{
Clinical and biological implications of CD133-positive and CD133-negative cells in glioblastomas
}

Kyeung Min Joo ${ }^{1,2,7}$, Shi Yean Kim¹,2,7 ${ }^{1}$ Xun Jin ${ }^{3}$, Sang Yong Song ${ }^{4}$, Doo-Sik Kong ${ }^{1}$, Jung-II Lee ${ }^{1}$, Ji Won Jeon ${ }^{1,2}$, Mi Hyun Kim ${ }^{1,2}$, Bong Gu Kang ${ }^{1,2}$, Yong Jung ${ }^{1,2}$, Juyoun Jin ${ }^{1,2}$, Seung-Chyul Hong ${ }^{1}$, Woong-Yang Park ${ }^{5}$, Dong-Sup Lee ${ }^{6}$, Hyunggee $\mathrm{Kim}^{3}$ and Do-Hyun Nam ${ }^{1,2}$

A number of recent reports have demonstrated that only CD133-positive cancer cells of glioblastoma multiforme (GBM) have tumor-initiating potential. These findings raise an attractive hypothesis that GBMs can be cured by eradicating CD133-positive cancer stem cells (CSCs), which are a small portion of GBM cells. However, as GBMs are known to possess various genetic alterations, GBMs might harbor heterogeneous CSCs with different genetic alterations. Here, we compared the clinical characteristics of two GBM patient groups divided according to CD133-positive cell ratios. The CD133low GBMs showed more invasive growth and gene expression profiles characteristic of mesenchymal or proliferative subtypes, whereas the CD133-high GBMs showed features of cortical and well-demarcated tumors and gene expressions typical of proneuronal subtype. Both CD133-positive and CD133-negative cells purified from four out of six GBM patients produced typical GBM tumor masses in NOD-SCID brains, whereas brain mass from CD133-negative cells showed more proliferative and angiogenic features compared to that from CD133-positive cells. Our results suggest, in contrast to previous reports that only CD133-positive cells of GBMs can initiate tumor formation in vivo CD133-negative cells also possess tumor-initiating potential, which is indicative of complexity in the identification of cancer cells for therapeutic targeting.

Laboratory Investigation (2008) 88, 808-815; doi:10.1038/labinvest.2008.57; published online 16 June 2008

KEYWORDS: cancer stem cell; CD133; clinical manifestation; gene expression profile; glioblastoma

A recent concept in brain tumor biology is that brain tumors arise from cancer stem cells (CSCs) that are CD133 positive $\left(\mathrm{CD} 133^{(+)}\right)$. It has been reported that a small number of $\mathrm{CD}_{133^{(+)}}$glioblastoma multiforme (GBM) cells are able to recapitulate the original tumor in vivo, whereas millions of CD133-negative $\left(\mathrm{CD}_{133^{(-)}}\right)$cells could not produce brain tumor masses. ${ }^{1-6}$ However, accumulating evidence suggests that $\mathrm{CD} 133^{(-)} \mathrm{GBM}$ cells can also regenerate heterogenous tumors in vivo, ${ }^{7,8}$ and generation of the huge and rapidly growing tumors by only $\mathrm{CD} 133^{(+)}$CSCs would be difficult because more than $50 \%$ of $\mathrm{GBM}$ patients have few $\mathrm{CD} 133^{(+)}$cells. ${ }^{9}$

As a majority of neurogenic astrocytes in the adult brain are not recognized by a CD133 antibody, ${ }^{8}$ it is likely that
CD133 might be newly expressed in GBM CSCs that are derived from $\mathrm{CD}_{13} 3^{(-)}$adult neural stem cells (NSCs) or terminally differentiated brain cells, such as astrocytes, neurons, and oligodendrocytes. Given that the gene expression profile is changed when GBM recurs after treatments, ${ }^{10}$ it is plausible that new CD133 expression may occur if the characteristics of CSCs are changed or if some CSCs are selected by treatment. Furthermore, the wide-range variation in $\mathrm{CD}_{133^{(+)}}$cell ratio $(0.1-50 \% \text { in GBM patients })^{1-6}$ also suggests the existence of other GBM CSCs that do not express CD133.

Therefore, we hypothesize that there are several kinds of CSCs in the tumor mass of GMB, and these diverse CSCs

\footnotetext{
'Department of Neurosurgery, Samsung Medical Center and Samsung Biomedical Research Institute, Sungkyunkwan University School of Medicine, Seoul, South Korea, ${ }^{2}$ Cancer Stem Cell Research Center, Samsung Medical Center and Samsung Biomedical Research Institute, Sungkyunkwan University School of Medicine, Seoul, South Korea; ${ }^{3}$ School of Life Sciences and Biotechnology, Korea University, Seoul, South Korea; ${ }^{4}$ Department of Pathology, Samsung Medical Center and Samsung Biomedical Research Institute, Sungkyunkwan University School of Medicine, Seoul, South Korea; ${ }^{5}$ Department of Biochemistry and Molecular Biology, Seoul National University College of Medicine, Seoul, South Korea and ${ }^{6}$ Department of Anatomy, Seoul National University College of Medicine, Seoul, South Korea

Correspondence: Dr Do-H Nam, MD, PhD, Department of Neurosurgery, Samsung Medical Center and Biomedical Research Institute, Sungkyunkwan University School of Medicine \#50, Irwon-dong, Gangnam-gu, Seoul 135-710, South Korea or Dr H Kim, PhD, Cell Growth Regulation Laboratory, School of Life Sciences and Biotechnology, Korea University, Anam-dong, Seongbuk-gu, Seoul 136-713, South Korea.

E-mail: nsnam@skku.edu or hg-kim@korea.ac.kr

${ }^{7}$ These authors contributed equally to this work.
}

Received 09 March 2008; revised 03 April 2008; accepted 03 April 2008 
possess diverse cellular and molecular characteristics, such as tumor initiation potential, chemo-radiation resistance, and gene expression profile.

\section{MATERIALS AND METHODS}

\section{Tumor Specimens and Primary Tumor Cultures}

Following informed consent, tumor samples classified as GBMs based on World Health Organization criteria ${ }^{11}$ were obtained from patients undergoing surgical treatment at the Samsung Medical Center (Seoul, Korea) in accordance with the appropriate Institutional Review Boards. Tumors were enzymatically dissociated into single cells and red blood cells were removed by differential centrifugation. Dissociated cells were cultured in the 'NBE' conditions consisting of NeuroBasal Media (Invitrogen, Carlsbad, CA, USA), N2 and B27 supplements (Invitrogen), as well as human recombinant bFGF and EGF ( $50 \mathrm{ng} / \mathrm{ml}$ each; R\&D Systems, Minneapolis, MN, USA). To differentiate cultured cells, cells were plated onto poly-L-lysine/laminin mixture-precoated culture dish (Invitrogen) and were subjected to growth in DMEM with $10 \%$ fetal bovine serum (10\% FBS/DMEM; Cambrex, East Rutherford, NJ, USA). Immunofluorescence assay was performed using following antibodies: Tuj1 (Chemicon, Billerica, MA, USA), GFAP (Sigma, St Louis, MN, USA), and O4 (Chemicon).

\section{Fluorescence-Activated Cell Sorting and Intracranial Cell Transplantation}

Dissociated GBM cells were labeled with $10 \mu \mathrm{l}$ anti-CD133/2PE antibody (Miltenyi Biotec, Bergisch Gladbach, Germany) per $10^{6}$ cells. $\mathrm{CD} 133^{(+)}$cell ratios were analyzed, and $\mathrm{CD} 133^{(+)}$and $\mathrm{CD} 133^{(-)}$populations were sorted using a FACSAria machine (BD Biosciences, San Jose, CA, USA). A total of $5 \times 10^{3}-2 \times 10^{4}$ purified cells suspended in $5 \mu \mathrm{l}$ HBSS were stereotactically injected into 6-week-old NOD/SCID mouse brains ( $2 \mathrm{~mm}$ left and $1 \mathrm{~mm}$ anterior to the bregma, $2 \mathrm{~mm}$ deep).

\section{Specimens and Immunohistochemistry}

As shown neurological symptom, mice were sacrificed and their brains were removed. The brains were processed for paraffin or frozen section. Paraffin sections were stained with hematoxylin and eosin as per standard histopathological technique and the tumor volume was recorded (largest width ${ }^{2} \times$ largest length $\times 0.5$ ). Frozen sections were fixed with acetone and then immunohistochemistry was performed as described previously ${ }^{12}$ using following antibodies: CD31 (BD Pharmingen, San Diego, CA, USA), PCNA (Dako, Glostrup, Denmark), and p-Akt (Cell signaling technology, Danvers, MA, USA).

\section{RNA Expression Array and Data Analysis}

Total RNAs were isolated using TRIZOL reagent (Invitrogen) and further purified using RNeasy Mini Kit (Qiagen, Hilden, Germany). Samples were processed and hybridized to
Affymetrix U-133 plus2 GeneChip Arrays according to the Affymetrix GeneChip Expression Analysis Technical Manual (Affymetrix, Santa Clara, CA, USA). All arrays were confirmed to be within acceptable minimal quality control parameters. The gene expression CEL files were normalized using Robust Multichip Averaging procedure and PM-MM difference model was used to obtain the expression values. The hierarchical cluster analysis was performed using $\mathrm{R}$ package software 2.6.0 using the Euclidean distance with complete linkage.

\section{RESULTS}

GBM Patients Divided into CD133-High and CD133-Low GBM Groups Show Different Clinical Characteristics

To evaluate our hypothesis, we first divided GBM patients into a CD133-high group $\left(\mathrm{CD} 133^{(+)}\right.$cell ratio $\left.\geq 3 \%, n=7\right)$ and a CD133-low group $\left(\mathrm{CD} 133^{(+)}\right.$cell ratio $\left.<3 \%, n=13\right)$ by fluorescence-activated cell sorting (FACS) analysis with anti-CD133 antibody (Supplementary Figure 1, Table 1). We analyzed the patients' clinical characteristics using MRI scan data (Figure 1a) to determine whether these groups possess similar or different clinical characteristics. As compared to CD133-high GBMs, CD133-low GBMs have tendency to be localized within the deeper structures of the brain and to show more invasive growth patterns and ventricle involvement (Figure 1b). However, there was no significant difference in sphere-forming capacity between cells derived from CD133-high and CD133-low GBMs, as cultured under 'NBE' conditions (NeuroBasal Media supplemented with N2, B27, EGF, and bFGF) (Table 1).

\section{CD133-Low and CD133-High GBMs Show Different Clinical Outcomes}

As CD133-low GBMs showed more invasive morphologies in MRI scan, we tested whether disease progression after treatment is different between CD133-high and CD133-low GBM patients. The rate of disease progression after chemotherapy and radiotherapy was relatively higher in the CD133-low GBMs (45.5\%) compared to CD133-high GBMs (28.6\%) (Figure 1c). Therefore, these results indicate that CD133-high and CD133-low GBM patients have different clinical characteristics and outcomes.

\section{CD133-Low and CD133-High GBMs Show Different Gene Expression Profiles}

Next, we compared gene expression patterns between subgroups of patients whose GBMs had the lowest and highest $\mathrm{CD}_{133^{(+)}}$cell ratios, termed 'CD133-lowest' $\left(\mathrm{CD} 133^{(+)}\right.$cell ratio $<0.5 \%, n=3)$ and 'CD133-highest' $\left(\mathrm{CD} 133^{(+)}\right.$cell ratio $>15 \%, n=4)$. Following cDNA microarray analysis, we performed a hierarchical clustering of 34 selected genes that are used for subclassification of GBM, such as proneuronal, mesenchymal, and proliferative subtypes. ${ }^{10,11}$ As shown in Figure 2a, CD133-lowest GBMs showed a relative upregulation of genes that have been previously linked to the 'mesenchymal' or 'proliferative' subtype, which has worse clinical outcome, whereas CD133-highest GBMs showed 
Table 1 Summary of patients and their clinical characteristics

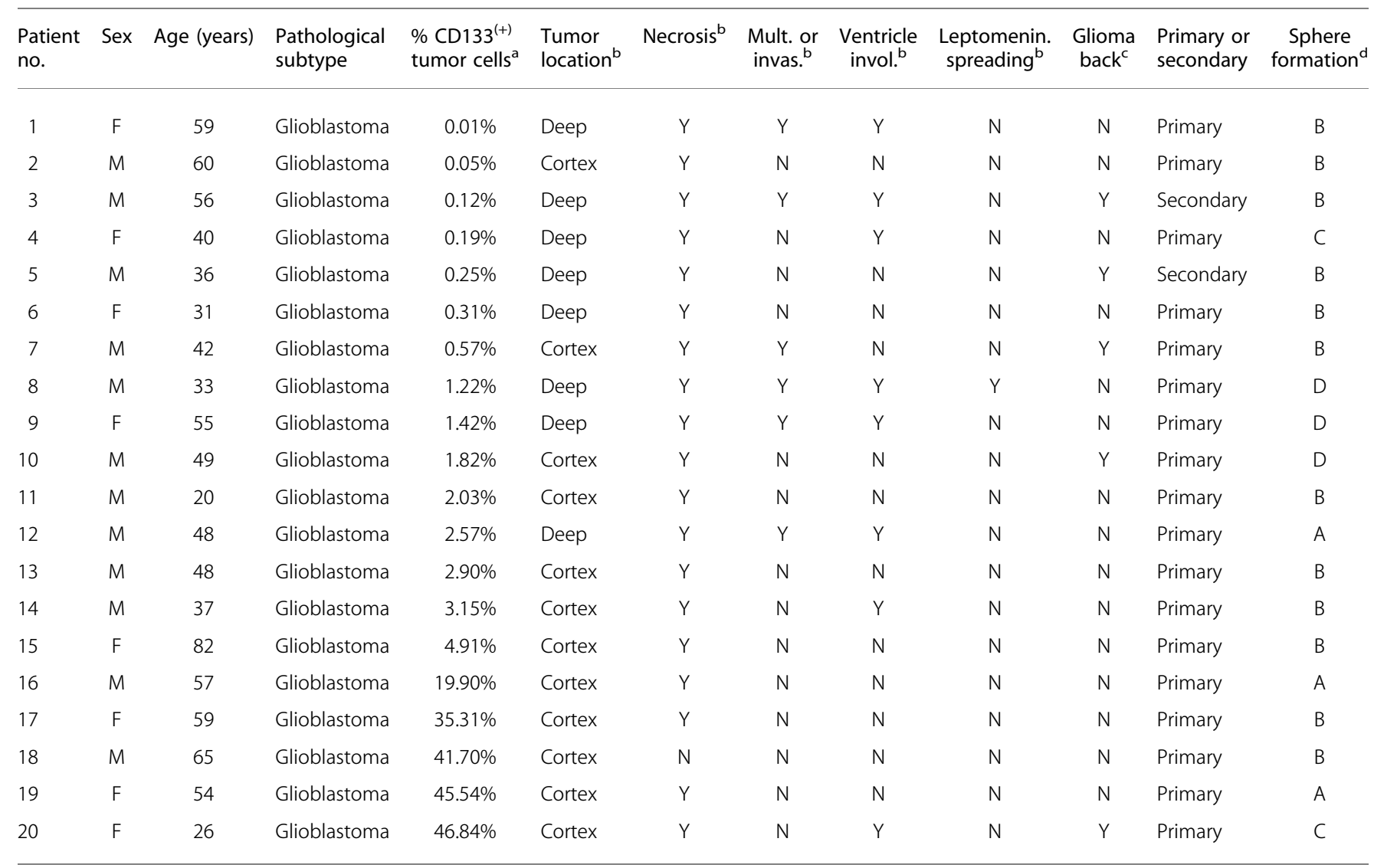

Cortex, cerebral cortex; deep, deep seated; glioma back., glioma background; invas., invasiveness; leptomenin. spreading, leptomeningeal spreading; mult., multiplicity of tumor mass; ventiricle invol., ventricle involvement.

${ }^{\mathrm{a}}$ Flow cytometry was used to determine the proportion of $\mathrm{CD} 133^{(+)}$cells from each patient's tumor mass.

${ }^{\mathrm{b}} \mathrm{MRI}$ scan data were used.

${ }^{\mathrm{C}}$ Pathologically diagnosed.

${ }^{\mathrm{d}}$ After $1 \times 10^{6}$ acutely dissociated cells were cultured under sphere-forming conditions for 14 days, the number of spheres was analyzed. A: $>50$; B: $11-50$; C: $1-10 ; \mathrm{D}: 0$.

higher expression of genes that are known to be upregulated in the 'proneuronal' GBM subtype. ${ }^{10}$ These results were further validated by real-time RT-PCR, showing that expression of DLL3 and SOX8 (proneuronal genes) is decreased, while YKL40 (a mesenchymal gene) was upregulated in the CD133-lowest GBMs (Figure 2b).

Previous data showed that when dissociated GBM cells are cultured in 'NBE' conditions, which were originally designed to culture normal NSCs, they become very similar to normal NSCs and thereby recapitulate the genotype, gene expression patterns, and in vivo biology of GBMs. ${ }^{13}$ Interestingly, when cells dissociated from CD133-high GBM patients (no. 16 or 19) $\left(\mathrm{CD}_{133^{(+)}}\right.$cell ratios $=19.90$ and $45.54 \%$, respectively) were subjected to growth in 'NBE' conditions for 10 days, expression of genes characteristic of the 'proneuronal' GBM subtype was markedly decreased, whereas expression of 'mesenchymal' or 'proliferative' GBM subtype-related genes increased (Figure 2c). However, both $\mathrm{CD}_{13} 3^{(+)}$and CD133 ${ }^{(-)}$GBM cells grown in 10\% FBS/DMEM conditions for 10 days were prone to differentiate into various brain cells that express GFAP (astrocyte), Tuj1 (neuron), or O4 (oligodendrocyte) (data not shown). These results indicate that the gene expression pattern of CSCs can change depending on in vivo and in vitro conditions, without alteration of their differentiation property.

\section{Both CD133-Low and CD133-High GBMs Show Stem Cell-Like Characteristics In Vitro}

We compared the sphere-forming abilities of the CD133-low or CD133-high GBMs in the 'NBE' condition and found that 9 in 13 CD133-low GBMs (69\%) made many spheres within a week (Figure 3a, Table 1). This frequency was comparable with that of CD133-high GBMs (six in seven, 86\%). Even the three CD133-lowest GBMs (patient nos. 1, 2, 3; CD133 ${ }^{(+)}$ cell ratio $<0.2 \%$ ) made spheres well in vitro (Table 1 ). Spheres formed by either CD133-low or CD133-high GBMs had similar morphology and size (Figure 3a). When the sphere cells were forced to differentiate in 10\% FBS/DMEM, 
a

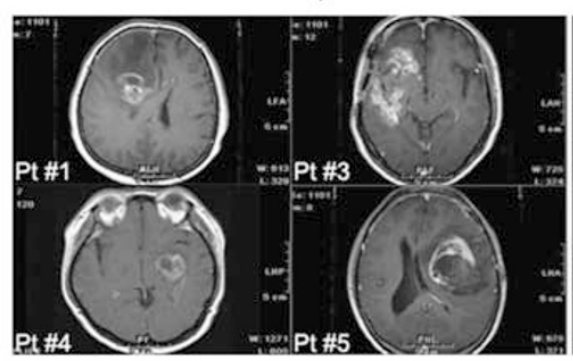

b

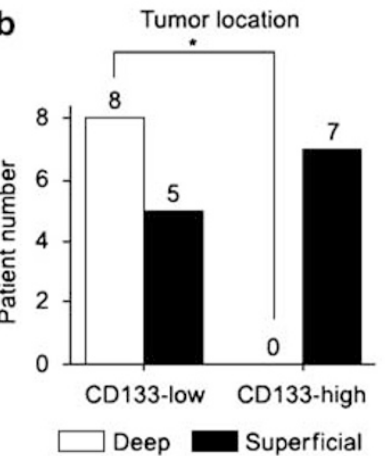

Invasiveness or multiplicity

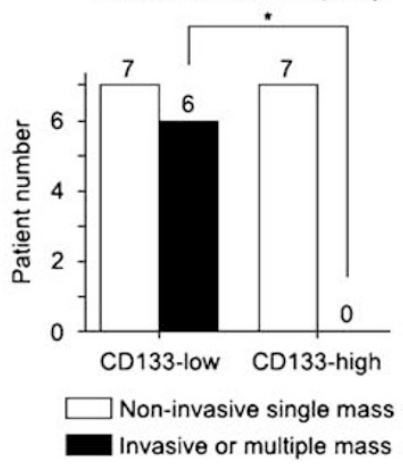

CD133-high patients

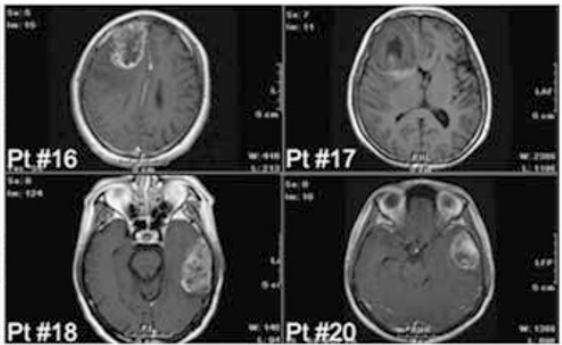

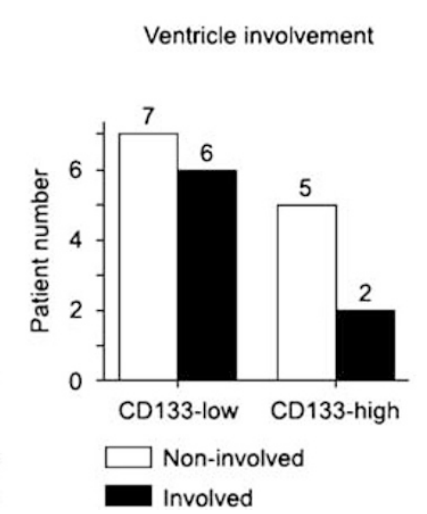

C Disease progression after treatment

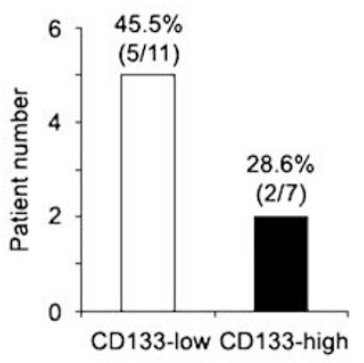

Figure 1 CD133-high and CD133-low GBM patient groups show different clinical characteristics. (a) Representative MRI scan data of four CD133-low patients (patient nos. 1, 3, 4, 5) and four CD133-high patients (patient nos. 16, 17, 18, 20) divided by CD133-positive cell ratio. (b) Comparisons of tumor location, invasiveness or multiplicity, and ventricle involvement between CD133-high patients and CD133-low patients. ${ }^{\star} P<0.05$.

(c) Comparison of disease progression rates between 11 CD133-low and seven CD133-high GBM patients after chemotherapy and radiotherapy.

a

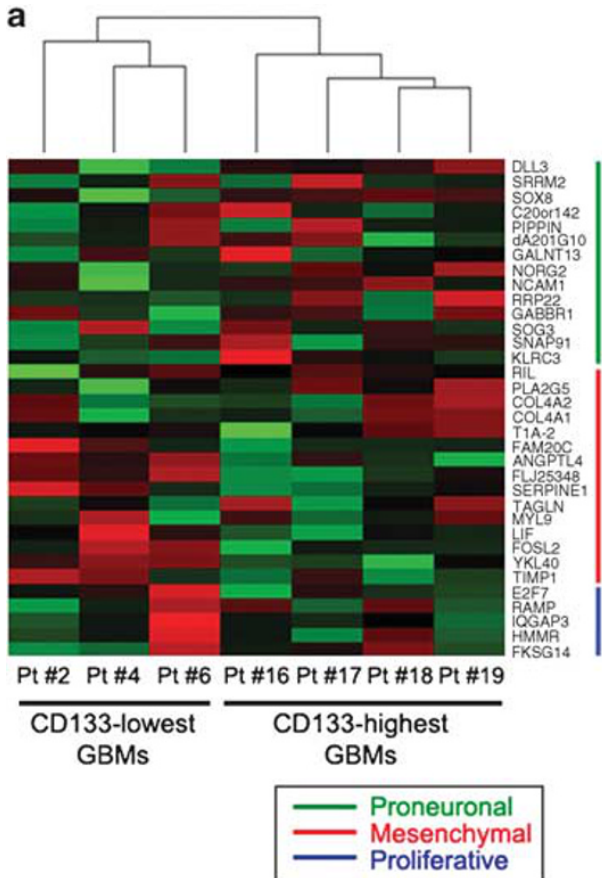

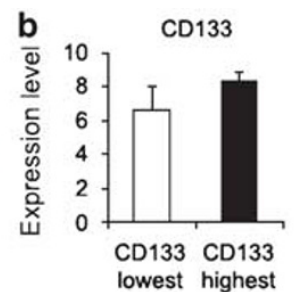

DLL3
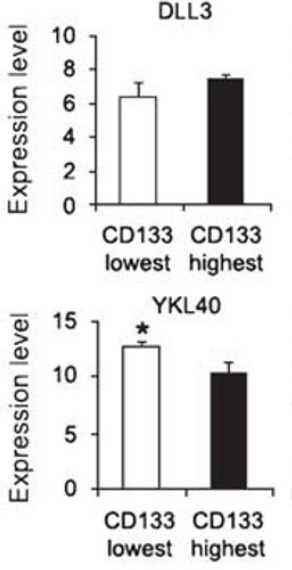

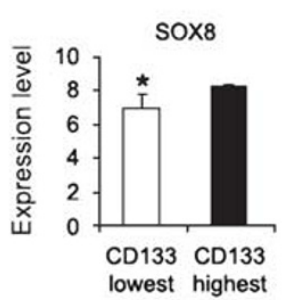

E2F7

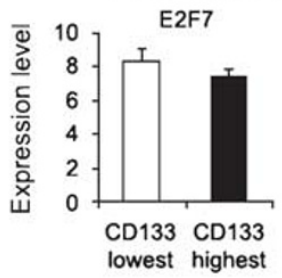

C

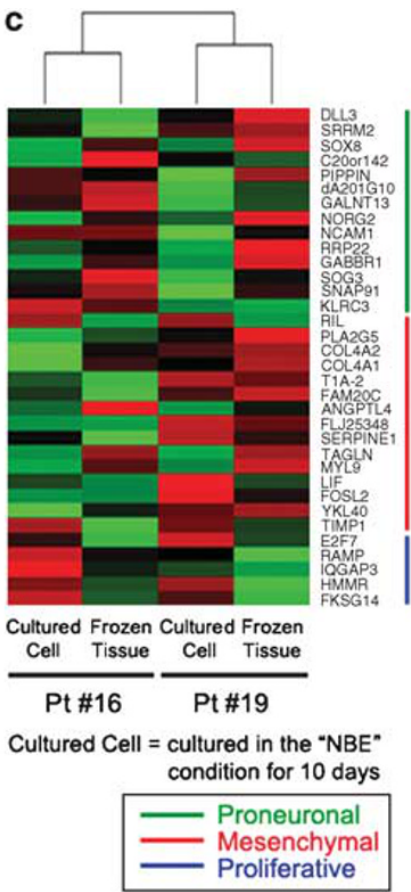

Figure 2 CD133-lowest and CD133-highest GBMs show different gene expression profiles. (a) Heatmaps showing expression profiles of the genes classified into 'proneuronal', 'mesenchymal', and 'proliferative' subtypes in three CD133-lowest and four CD133-highest GBM tumor masses. (b) The expression levels of five selected genes (CD133, DLL3, SOX8, YKL40, and E2F7) in three CD133-lowest and four CD133-highest GBM tumor masses were validated by real-time RT-PCR. ${ }^{*} P<0.05$. (c) Heatmaps showing the expression profiles of the genes classified into 'proneuronal', 'mesenchymal', and 'proliferative' subtypes in two CD133-high GBM tumor masses (patient nos. 16 and 19) and their cognate cells, which are cultured in 'NBE' conditions for 10 days after acute dissociation from tumor masses. 


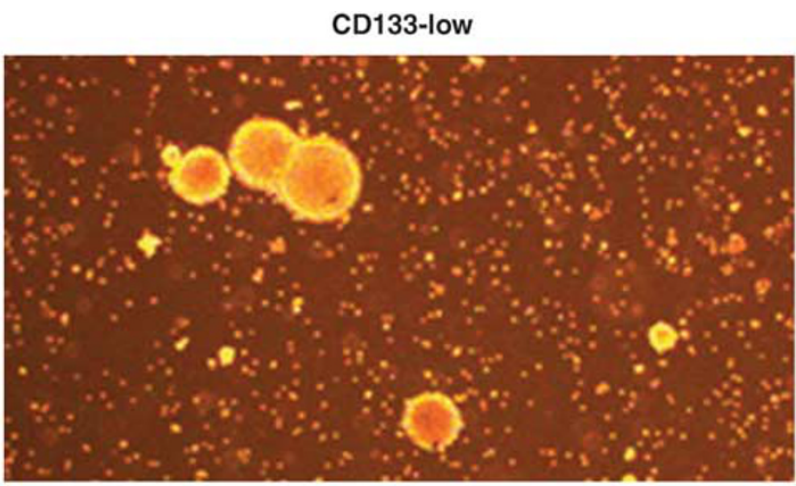

CD133-high

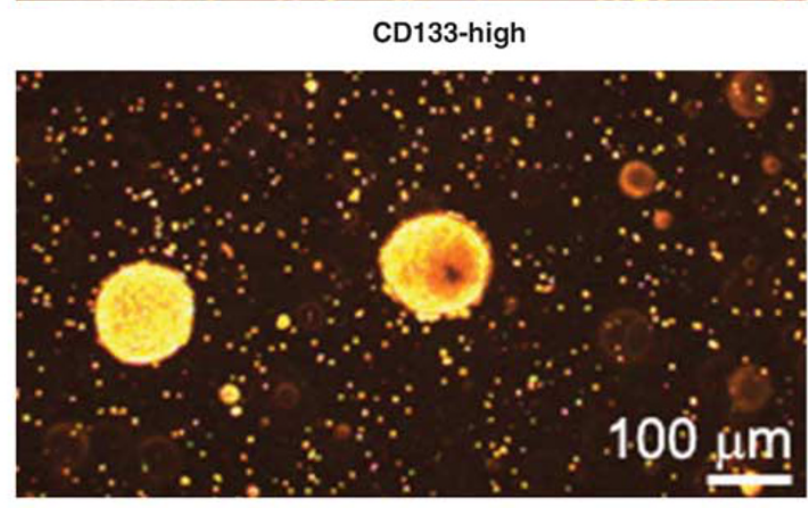

b

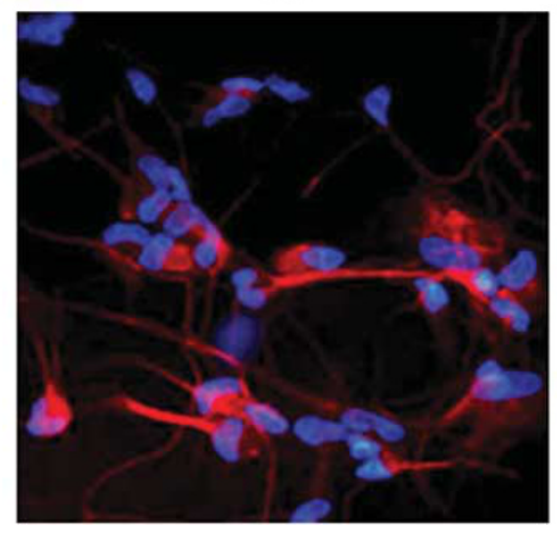

6

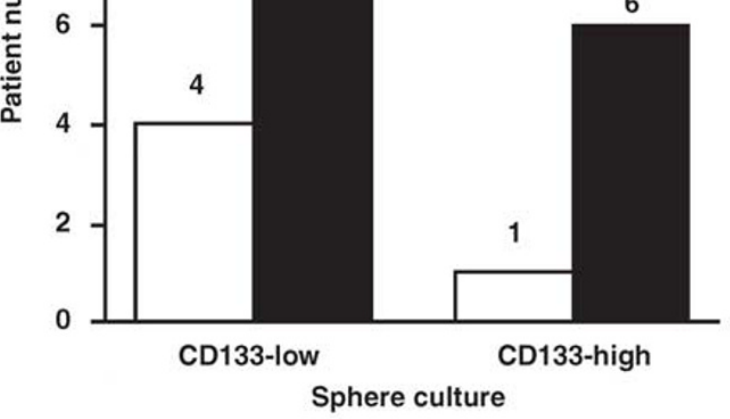

Poor neurosphere formation $>10$ days or more

Good neurosphere formation within a week
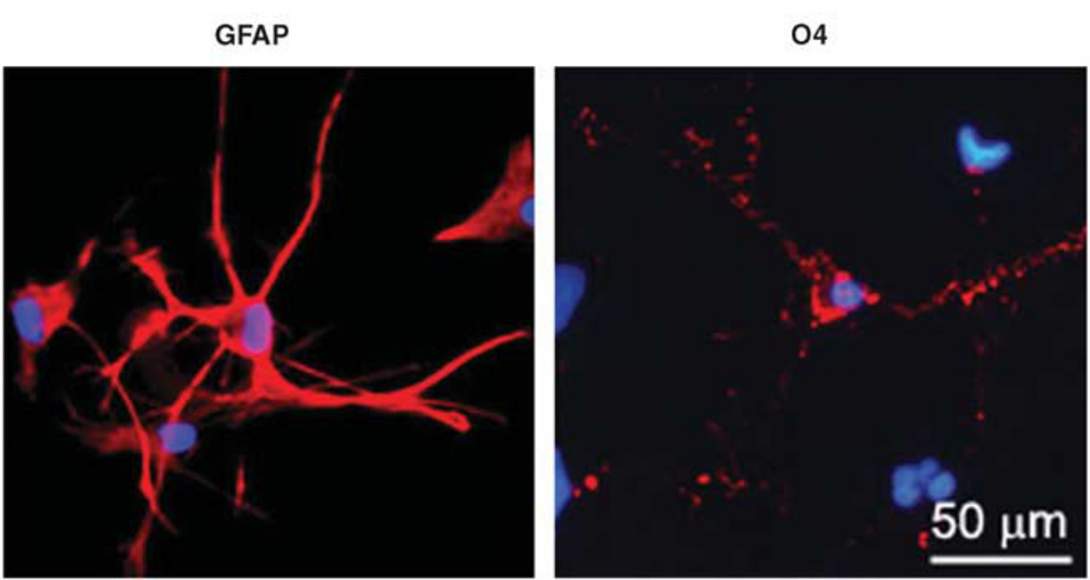

Figure 3 Both CD133-low and CD133-high GBMs show stem cell-like characteristics in vitro. (a) Neurosphere-forming ability in the 'NBE' condition was compared between cells dissociated from CD133-high and CD133-low GBMs. The representative photos of spheres derived from CD133-high and CD133-low GBMs (left panel). (b) Sphere cells cultured in the 'NBE' condition were differentiated with 10\% FBS. Representative photos present expressions of Tuj1, GFAP, and $\mathrm{O} 4$ determined by immunofluorescence assay.

most cells from both CD133-low and CD133-high GBMs showed morphology of differentiated cells and expressed differentiated neural cell markers such as Tuj1, GFAP, and O4 (Figure 3b; a representative data from GBM patient no. 12). These results suggest that cells from CD133-low GBMs also have stem cell-like characteristics in vitro.

\section{Both $\mathrm{CD} 133^{(-)}$and $\mathrm{CD} 133^{(+)}$GBM Cells Possess Tumor-Initiating Potential}

As it has been recently documented that some GBM patients have $\mathrm{CD} 133^{(-)} \mathrm{CSCs}$ and others have $\mathrm{CD} 133^{(+)},{ }^{7}$ and lowgrade gliomas possess multiple and spatially distinct clonal populations, ${ }^{14}$ we isolated $\mathrm{CD} 133^{(+)}$and $\mathrm{CD} 133^{(-)}$cells from tumor specimens of a number of GBM patients using FACS analysis (Figure 4a; a representative data from GBM patient no. 4) to compare tumor-initiating potential. When $\mathrm{CD}_{133^{(+)}}$or $\mathrm{CD} 133^{(-)}$cells purified by FACS sorting were injected into the brains of NOD/SCID mice $(n=3-5)$, tumors were produced in about half of the injected brains, indicating that both cell types harbor tumor-initiating potential $\left(\mathrm{CD} 133^{(+)}=7\right.$ in $17, \mathrm{CD} 133^{(-)}=11$ in 20$)$. In addition, both $\mathrm{CD} 133^{(+)}$and $\mathrm{CD} 133^{(-)}$cells purified from four out of six human GBM patients were able to generate tumor masses that show similar GBM histological features 
a

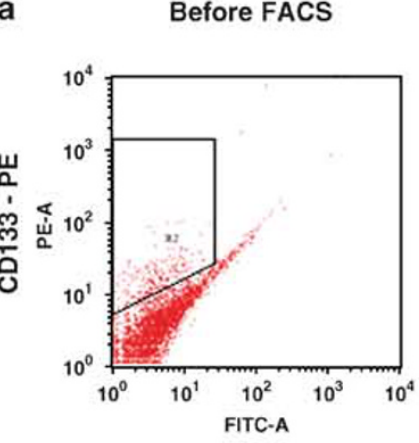

$\mathrm{CD}_{133^{(+)}}=2.96 \%$

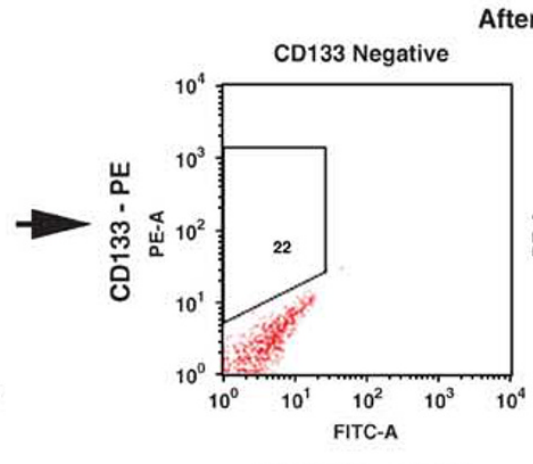

$\mathrm{CD}^{133^{(+)}}=0.00 \%$

fter FACS

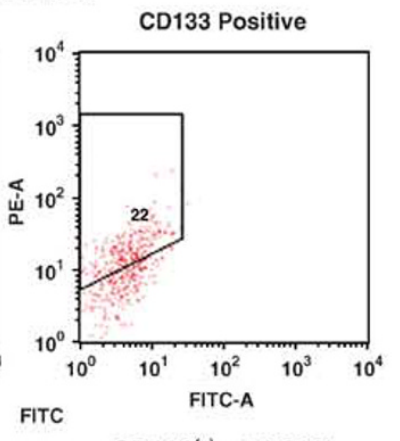

$C D 133^{(+)}=53.98 \%$

PCNA

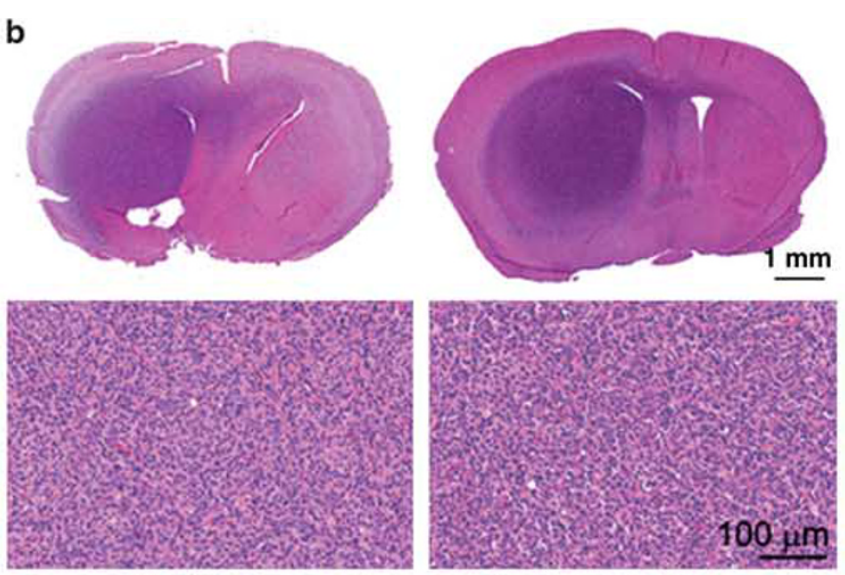

CD133 Negative

CD133 Positive c CD31

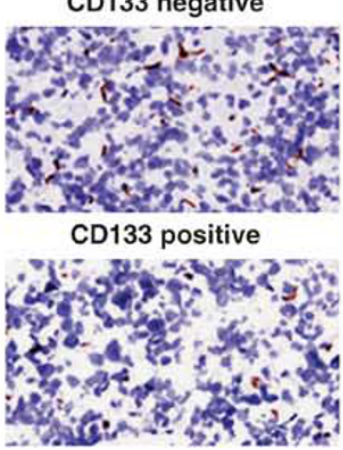

CD133 negative

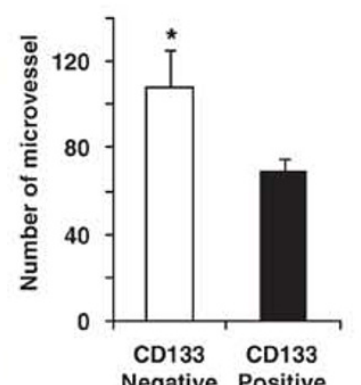

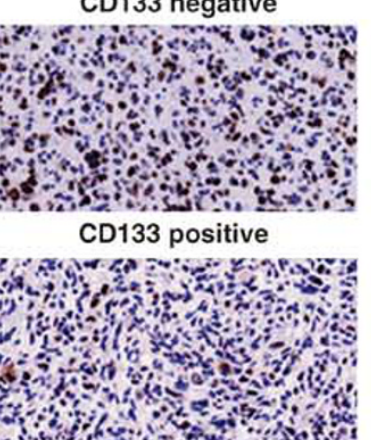

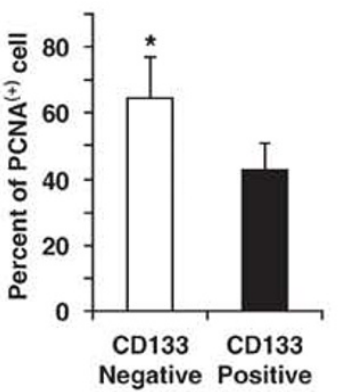

CD133 negative

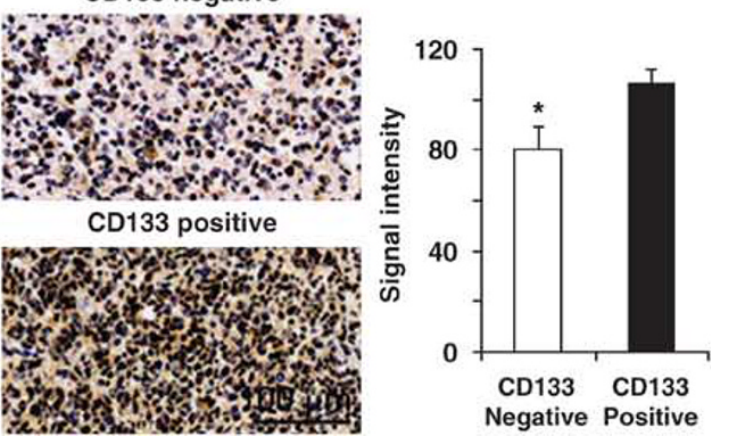

Figure 4 Both $\mathrm{CD} 133^{(-)}$and $\mathrm{CD} 133^{(+)} \mathrm{GBM}$ cells possess tumor-initiating potential. (a) Representative FACS data showing CD133 $3^{(+)}$and $\mathrm{CD} 133^{(-)}$cell populations isolated from one of $20 \mathrm{GBM}$ patients using FACS. (b) When orthotopically injected into NOD/SCID mice, both CD133 ${ }^{(+)}$and $C D 133^{(-)}$cells derived from one GBM patient produced tumor masses. Representative photos showing brain tumor tissue sections stained with hematoxylin and eosin. (c) Representative photos showing CD31-positive, PCNA-positive, and pAkt-positive cells in the tumor masses derived from $\mathrm{CD} 133^{(-)}$and $\mathrm{CD} 133^{(+)}$cells. Quantitative data showing microvessel numbers, $\mathrm{PCNA}{ }^{(+)}$and $\mathrm{pAkt}{ }^{(+)}$cell numbers in tumors derived from $\mathrm{CD} 133^{(-)}$and $\mathrm{CD} 133^{(+)}$cells. ${ }^{\star P}<0.05$. 


\begin{tabular}{|c|c|c|c|c|c|}
\hline $\begin{array}{l}\text { Patient } \\
\text { no. }\end{array}$ & $\begin{array}{l}\text { Sorting } \\
\text { method }\end{array}$ & $\begin{array}{l}\text { Injected cell } \\
\text { number }\end{array}$ & $\begin{array}{l}\text { Tumor } \\
\text { incidence }\end{array}$ & $\begin{array}{l}\text { Tumor volume } \\
\qquad\left(\mathrm{mm}^{3}\right)\end{array}$ & $\begin{array}{l}\text { First neurological } \\
\text { symptom (days) }\end{array}$ \\
\hline \multirow[t]{2}{*}{1} & FACS & $\mathrm{CD} 133^{(+)}=1 \times 10^{4}$ & $\mathrm{CD} 133^{(+)}=1 / 4$ & $\mathrm{CD} 133^{(+)}=2.2$ & $\mathrm{CD} 133^{(+)}=192$ \\
\hline & & $\mathrm{CD} 133^{(-)}=1 \times 10^{4}$ & $\mathrm{CD} 133^{(-)}=1 / 3$ & $\mathrm{CD} 133^{(-)}=6.6$ & $\mathrm{CD} 133^{(-)}=239$ \\
\hline & & $\mathrm{CD} 133^{(-)}=5 \times 10^{3}$ & $\mathrm{CD} 133^{(-)}=0 / 3$ & & \\
\hline \multirow[t]{2}{*}{4} & FACS & $\mathrm{CD} 133^{(+)}=1 \times 10^{3}$ & $\mathrm{CD} 133^{(+)}=1 / 2$ & $\mathrm{CD} 133^{(+)}=42.8$ & $\mathrm{CD}_{133^{(+)}}=139$ \\
\hline & & $\mathrm{CD} 133^{(-)}=2 \times 10^{4}$ & $\mathrm{CD} 133^{(-)}=4 / 5$ & $\mathrm{CD} 133^{(-)}=35.7 \pm 9.9$ & $\mathrm{CD} 133^{(-)}=123.7 \pm 13.3$ \\
\hline \multirow[t]{2}{*}{7} & FACS & $\mathrm{CD} 133^{(+)}=5 \times 10^{3}$ & $\mathrm{CD} 133^{(+)}=0 / 3$ & - & - \\
\hline & & $\mathrm{CD} 133^{(-)}=2 \times 10^{4}$ & $\mathrm{CD} 133^{(-)}=0 / 3$ & & \\
\hline \multirow[t]{2}{*}{18} & FACS & $\mathrm{CD} 133^{(+)}=2 \times 10^{4}$ & $\mathrm{CD} 133^{(+)}=2 / 2$ & $\mathrm{CD} 133^{(+)}=113.3 \pm 18.4$ & $\mathrm{CD} 133^{(+)}=90.5 \pm 5.0$ \\
\hline & & $\mathrm{CD} 133^{(-)}=2 \times 10^{4}$ & $\mathrm{CD} 133^{(-)}=3 / 3$ & $\mathrm{CD} 133^{(-)}=80.7 \pm 16.0$ & $\mathrm{CD} 133^{(-)}=120.7 \pm 23.0$ \\
\hline
\end{tabular}

FACS, fluorescence-activated cell sorting; GBM, glioblastoma multiforme.

(Table 2, Figure $4 \mathrm{~b}$ ). Furthermore, tumor volumes in the mice injected with sorted $\mathrm{CD} 133^{(+)}$or $\mathrm{CD} 133^{(-)}$cells were not significantly different, and neurological symptoms were detected at almost the same time point from injections (Table 2). However, although the volume and overall histology of tumor masses produced by injection of sorted $\mathrm{CD} 133^{(+)}$or $\mathrm{CD} 133^{(-)}$cells were relatively similar, the tumor masses from $\mathrm{CD}_{133^{(-)}}$cells contained more microvessels and $\mathrm{PCNA}^{(+)}$-proliferating cells (Figure 4c), whereas those from $\mathrm{CD} 133^{(+)}$cells were found to have significantly higher levels of phosphorylated Akt (pAkt, Figure 4c). These results suggest that there are multiple kinds of tumor-initiating cells, such as $\mathrm{CD} 133^{(-)}$and $\mathrm{CD} 133^{(+)}$ cells, in every GBM patient.

\section{DISCUSSION}

A new paradigm in brain tumor biology that only $\mathrm{CD} 133^{(+)}$ cancer cells harbor a tumor-initiating potential raises an attractive hypothesis that GBMs can be cured if one eradicates CD133 ${ }^{(+)}$CSCs, which occupy small portion of GMB cells. ${ }^{3,4}$ However, the story would not be simple, as GBMs have been known to possess various genetic alterations and to consist of heterogeneous cell populations, ${ }^{15,16}$ arguing that CSCs in GBMs might also be heterogeneous and possess different genetic alterations. In support of above postulation, it has been recently documented that some GBM patients have $\mathrm{CD} 133^{(-)} \mathrm{CSC}$ and others have $\mathrm{CD} 133^{(+)}{ }^{7}$ Even lowgrade gliomas, which show more relatively homogeneous histology than GBMs, have been reported to possess multiple and spatially distinct clonal populations. ${ }^{14}$ Furthermore, in the present study, we demonstrated that there are CSCs in both $\mathrm{CD} 133^{(+)}$and $\mathrm{CD} 133^{(-)}$cell population originated from one GBM patient, and both $\mathrm{CD} 133^{(-)}$and $\mathrm{CD} 133^{(+)}$ cells enabled the formation of tumor masses that showed similar volumes at 3 months post-injections.

Consistent with a previous report that GBM patients can be divided into groups that present different prognosis, ${ }^{10}$ we found that GBM patients can be classified into two groups (CD133-low and CD133-high group) according to their $\mathrm{CD}_{133^{(+)}}$cell ratios, and CD133-low GBMs $\left(\mathrm{CD} 133^{(+)}\right.$cell ratio $<3 \%$ ) showed more aggressive morphologies as determined by MRI scan and unique gene expression patterns ('mesenchymal' or 'proliferative' subtype) that are related to worse prognosis. ${ }^{10,11}$ It is interesting to note that it has been reported that gene expression profiles change from the 'proneuronal' to 'mesenchymal' subtype in GBMs recurred after treatment. ${ }^{10}$ As $\mathrm{CD} 133^{(-)}$CSCs represented the 'mesenchymal' type, it might be plausible that $\mathrm{CD} 133^{(-)}$CSCs might be more resistant to radiotherapy and chemotherapy compared to $\mathrm{CD} 133^{(+)}$CSCs, which are already recognized to be markedly resistant to conventional anticancer therapies. ${ }^{17,18}$

When GBM cells dissociated from GBM patients are cultured in the 'NBE' condition, which is originally designed to culture the normal NSCs, they harbor extensive similarities to normal NSCs and thereby recapitulate the genotype, gene expression patterns, and in vivo biology of GBMs. ${ }^{13}$ As GBM cells dissociated from CD133-highest $\mathrm{GBMs}\left(\mathrm{CD} 133^{(+)}\right.$cell ratios $=19.90$ or $45.54 \%$ ), which presented 'proneuronal' subtype of gene expression, were cultured in the ' $\mathrm{NBE}$ ' condition for 10 days, their gene expression patterns were changed from 'proneuronal' subtype to 'mesenchymal' or 'proliferative' subtypes that were observed from $\mathrm{CD} 133^{(-)}$ CSCs, arguing that $\mathrm{CD} 133^{(-)}$CSCs might have advantages for survival or proliferation in the 'NBE' condition.

Taken together, our data show that there are at least two kinds of CSCs $\left(\mathrm{CD}_{133^{(+)}}\right.$and $\left.\mathrm{CD} 133^{(-)}\right)$in each GBM 
patient and that they have different biological and clinical characteristics. An understanding of all GBM CSC types and their clinical implications, as well as development of new GBM CSC-specific markers, should be a crucial step toward more effective therapeutic modalities against human GBMs.

Supplementary Information accompanies the paper on the Laboratory Investigation website (http://www.laboratoryinvestigation.org)

\section{ACKNOWLEDGEMENTS}

This work was supported by the Korea Science and Engineering Foundation (KOSEF) grant funded by the Korea government (MOST) (no.

MM10641000104-06N4100-1040 to DH Nam) and a grant from the National R\&D Program for Cancer Control, Ministry of Health \& Welfare, Republic of Korea (0720030 to H Kim).

1. Reya T, Morrison SJ, Clarke MF, et al. Stem cells, cancer, and cancer stem cells. Nature 2001;414:105-111.

2. Pardal R, Clarke M, Morrison S. Applying the principles of stem-cell biology to cancer. Nat Rev Cancer 2003;3:895-902.

3. Singh SK, Hawkins C, Clarke ID, et al. Identification of human brain tumour initiating cells. Nature 2004;432:396-401.

4. Galli $R$, Binda $E$, Orfanelli $U$, et al. Isolation and characterization of tumorigenic, stem-like neural precursors from human glioblastoma. Cancer Res 2004;64:7011-7021.

5. Hemmati HD, Nakano I, Lazareff JA, et al. Cancerous stem cells can arise from pediatric brain tumors. Proc Natl Acad Sci USA 2003;100:15178-15183.

6. Yuan X, Curtin J, Xiong Y, et al. Isolation of cancer stem cells from adult glioblastoma multiforme. Oncogene 2004;23:9392-9400.

7. Beier D, Hau P, Proescholdt M, et al. CD133(+) and CD133(-) glioblastoma-derived cancer stem cells show differential growth characteristics and molecular profiles. Cancer Res 2007;67: 4010-4015.

8. Pfenninger CV, Roschupkina $T$, Hertwig $F$, et al. CD133 is not present on neurogenic astrocytes in the adult subventricular zone, but on embryonic neural stem cells, ependymal cells, and glioblastoma cells. Cancer Res 2007;67:5727-5736.

9. Sakariassen $P \emptyset$, Immervoll $H$, Chekenya M. Cancer stem cells as mediators of treatment resistance in brain tumors: status and controversies. Neoplasia 2007;9:882-892.

10. Phillips HS, Kharbanda S, Chen R, et al. Molecular subclasses of high-grade glioma predict prognosis, delineate a pattern of disease progression, and resemble stages in neurogenesis. Cancer Cell 2006;9:157-173.

11. Kleihues P, Louis DN, Scheithauer BW, et al. The WHO classification of tumors of the nervous system. J Neuropathol Exp Neurol 2002;61: 215-225.

12. Nam DH, Park K, Park C, et al. Intracranial inhibition of glioma cell growth by cyclooxygenase-2 inhibitor celecoxib. Oncol Rep 2004;11:263-268.

13. Lee J, Kotliarova $S$, Kotliarov $Y$, et al. Tumor stem cells derived from glioblastomas cultured in bFGF and EGF more closely mirror the phenotype and genotype of primary tumors than do serum-cultured cell lines. Cancer Cell 2006;9:391-403.

14. Coons SW, Johnson PC, Shapiro JR. Cytogenetic and flow cytometry DNA analysis of regional heterogeneity in a low grade human glioma. Cancer Res 1995;55:1569-1577.

15. Wiltshire RN, Rasheed BK, Friedman HS, et al. Comparative genetic patterns of glioblastoma multiforme: potential diagnostic tool for tumor classification. Neuro Oncol 2000;2:164-173.

16. Ohgaki H, Kleihues P. Genetic pathways to primary and secondary glioblastoma. Am J Pathol 2007;170:1445-1453.

17. Bao $\mathrm{S}, \mathrm{Wu} \mathrm{Q}, \mathrm{McL}$ endon $\mathrm{RE}$, et al. Glioma stem cells promote radioresistance by preferential activation of the DNA damage response. Nature 2006;444:756-760.

18. Liu G, Yuan X, Zeng Z, et al. Analysis of gene expression and chemoresistance of CD133+ cancer stem cells in glioblastoma. Mol Cancer 2006;5:67. 